\title{
Spontaneous Pneumomediastinum in Covid-19 Pneumonia: Two Cases and Review of the Literature
}

\author{
(1) Hasan Kara', Aysegul Bayir', (1) Selim Değirmenci'2, Huseyin Yildiran³, (1) Ahmet Ak' \\ 'Selçuk University, Faculty of Medicine, Department of Emergency Medicine, Konya, Turkey \\ 2 Bilecik Training and Research Hospital, Department of Emergency Medicine, Bilecik, Turkey. \\ ${ }^{3}$ Selçuk University, Faculty of Medicine, Department of Thoracic Surgery, Konya, Turkey.
}

\begin{abstract}
Spontaneous pneumomediastinum (PM) is a rare presentation associated with Coronavirus disease 2019 (COVID-19). Here, we report two cases presented with spontaneous PM associated with COVID-19 pneumonia.A 58-year-old female patient was admitted to the Emergency Department (ED) with dyspnea, hoarseness, myalgia, and cough. A chest CT scan revealed subcutaneous emphysema, diffuse PM, bilateral diffuse areas of ground-glass opacity, and consolidations. The patient was transferred to the critical care unit. In critical intensive care, the patient developed multi-organ failure and expired on the 10th day. A 34-year-old male patient admitted to the ED with dyspnea and cough. A chest CT scan revealed diffuse PM, bilateral diffuse areas of ground-glass opacity, and consolidations. The patient was transferred to the critical care unit. His progress was uneventful and he was discharged after 12 days. Patients suspected of PM should be examined closely during the differential diagnosis of chest pain, dyspnea, subcutaneous emphysema, and various lung-and heart-induced states. Patients diagnosed with spontaneous PM should be hospitalized for observation because the condition can be associated with complications, including death.

Key Words: COVID-19, Dyspnea, Pneumomediastinum, Complication
\end{abstract}

\section{Introduction}

Coronavirus disease 2019 (COVID-19) is an infectious disease caused by severe acute respiratory syndrome coronavirus 2 (SARS-CoV-2), which was first identified in Wuhan, China, in 2019. Pneumomediastinum (PM), also known as mediastinal emphysema, is defined as the presence of air or other gas in the mediastinum. PM can be classified as traumatic, spontaneous, or iatrogenic. Spontaneous PM occurs due to increased alveolar pressure, associated with coughing, vomiting, straining, and the Valsalva maneuver, resulting in the spontaneous rupture of alveoli. Spontaneous PM has been associated with asthma, chronic obstructive pulmonary disease, mediastinal infections, mechanical ventilation, excessive exercise, cannabis or cocaine intake, and diffuse interstitial fibrosis ${ }^{1}$. Spontaneous and traumatic PM symptoms are nearly identical, with the most common symptom being chest pain that begins acutely and is localized behind the sternum. The clinical presentation of PM can range from non-specific symptoms to life-threatening respiratory failure. Subcutaneous emphysema in the neck and chest and Hamman's sign during heart auscultation on physical examination can also be caused by serious cardiovascular collapse ${ }^{2,3}$. Here, we present the clinical cases of two patients who tested positive for COVID-19 and presented with spontaneous PM and discuss the possible mechanisms underlying this association.

\section{Case Reports}

Case 1

A 58-year-old female patient with a past medical history of asthma presented to the Emergency Department (ED) with dyspnea, hoarseness, myalgia, and cough, lasting for five days. The patient's vital signs were as follows: blood pressure was 120/80 mmHg; tachycardic heart rate of 105 bpm; mild tachypnea, with a respiratory rate of 20 breaths per minute; temperature was $36.2{ }^{\circ} \mathrm{C}$; and transcutaneous oxygen saturation was $84 \%$ on room air. Physical examination revealed decreased breath sounds, bilaterally, and soft tissue crepitus in the bilateral clavicle, sternum, and anterior cervical area. Auscultation of the heart revealed a loud crunch-like sound during systole, consistent with "Hamman's crunch." The patient's abdominal examination was normal, as were skin, neurological, and psychiatric evaluations. Routine laboratory tests, cardiac enzymes, and electrocardiogram (ECG) were normal. The RT-PCR analysis for COVID-19 was positive 10 days prior to presentation, and the patient was treated with favipiravir for 5 days. The initial laboratory tests revealed an elevated C-reactive protein concentration of $198 \mathrm{mg} / \mathrm{L}$ (reference: $<8 \mathrm{mg} / \mathrm{L}$ ). Complete blood count showed a leukocyte count of $8.0 \times$ 109/L (reference: $3.5-1.5 \times 109 / \mathrm{L}$ ), with a relative left shift (9\% band neutrophils [reference: $<37 \%$ ]) and lymphopenia
Corresponding Author: Hasan Kara e-mail: hasankara42@gmail.com Received: 28.11 .2021 - Accepted: 30.12 .2021

DOI: $10.33706 /$ jemcr.1015741

OCopyright 2020 by Emergency Physicians Association of Turkey - Available online at www.jemcr.com
Cite this article as: Kara $\mathrm{H}$, Bayir A, Degirmenci $\mathrm{S}$, Yildiran $\mathrm{H}, \mathrm{Ak}$ A. Spontaneous pneumomediastinum in covid-19 pneumonia: two cases and review of the literature. Journal of Emergency Medicine Case Reports. 2022;13(1): 34-36 
(5\% [reference: 10\%-50\%]). Serum levels of D-dimer and ferritin were both elevated, at 1,082 $\mu \mathrm{g} / \mathrm{L}$ (reference: $<500$ $\mu \mathrm{g} / \mathrm{L}$ ) and 2,636 $\mu \mathrm{g} / \mathrm{L}$ (reference: 23.9-336.2 $\mu \mathrm{g} / \mathrm{L}$ ), respectively. The ECG showed sinus tachycardia at a rate of 105 bpm without signs of ischemia. The posteroanterior chest radiograph showed bilateral pulmonary infiltrates, PM, and subcutaneous emphysema into the neck. A chest CT scan revealed subcutaneous emphysema, diffuse PM, bilateral diffuse areas of ground-glass opacity, and consolidations, which are all features compatible with COVID-19 pneumonia (Figure 1). No evidence of pneumothorax, pneumopericardium, rib fractures, or tracheal or bronchial injuries was observed. Management was conservative and consisted primarily of supportive care. The patient was transferred to the critical care unit for monitoring and the regulation of medical treatment. The patient was started on anti-infective therapy, with $4,5 \mathrm{~g}$ of piperacillin/tazobactam three times a day and $400 \mathrm{mg}$ moxifloxacin once a day, low-dose intravenous methylprednisolone $(1 \mathrm{mg} / \mathrm{kg}$ ) following $1 \mathrm{~g}$ of Methylprednisolone for three days, enoxaparin sodium for therapeutic anticoagulation, convalescent plasma therapy $250 \mathrm{cc}$ for two times, and symptomatic supportive therapy, which was adjusted according to clinical conditions. The patient was hemodynamically stable, and high-flow nasal cannula oxygen therapy was attempted. The fraction of inspired oxygen (FiO2) concentration and oxygen flow rate were $100 \%$ and $60 \mathrm{~L} / \mathrm{min}$, respectively. In critical intensive care, the patient developed multi-organ failure, requiring mechanical ventilation and vasopressor support. On the 10th day, the patient expired due to cardiopulmonary arrest.
Case 2

A 34-year-old male patient with a past medical history of cerebral palsy presented to the ED with dyspnea and cough, lasting for nine days. The blood pressure was 130/75 mm $\mathrm{Hg}$, pulse was 98 beats per minute, respiratory rate was 16 breaths per minute, temperature was $36.5^{\circ} \mathrm{C}$, and transcutaneous oxygen saturation was $91 \%$ on room air. The breath sounds were normal and equal in both lungs. Routine laboratory tests, cardiac enzymes, and ECG were normal. The RT-PCR analysis for COVID-19 was positive 9 days prior to presentation, and the patient was treated with favipiravir for 5 days. The initial laboratory tests revealed an elevated C-reactive protein concentration of 71,3 mg/L. Complete blood count was completely normal. Serum levels of D-dimer and ferritin were both elevated, at $1,656 \mu \mathrm{g} / \mathrm{L}$ and $5,483 \mu \mathrm{g} / \mathrm{L}$, respectively. A chest $\mathrm{CT}$ scan revealed diffuse PM, bilateral diffuse areas of ground-glass opacity, and consolidations, which are all features compatible with COVID-19 pneumonia (Figure 2). The patient was transferred to the critical care unit for monitoring and the regulation of medical treatment. The patient was started on anti-infective therapy, with 400 mg moxifloxacin once a day, low-dose intravenous methylprednisolone (40 mg/day), enoxaparin sodium for therapeutic anticoagulation, and symptomatic supportive therapy, which was adjusted according to clinical conditions. The patient was hemodynamically stable, and high-flow nasal cannula oxygen therapy was attempted. His progress was uneventful and he was discharged after 12 days.

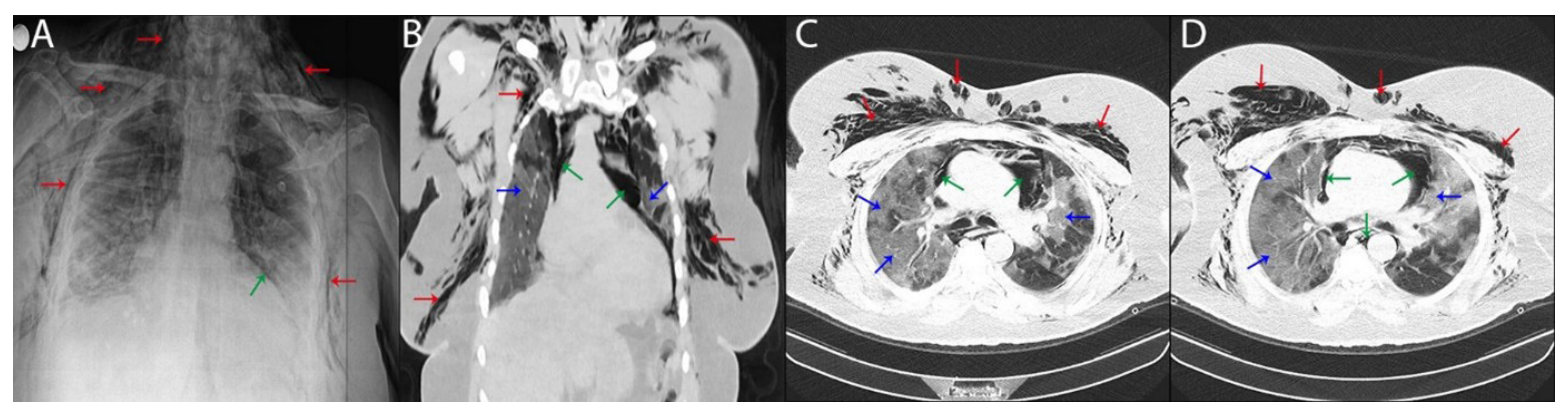

Figure 1: Radiological findings of Case 1. The posteroanterior chest radiograph (A) showed PM (green arrow), and subcutaneous emphysema (red arrow) into the neck. A coronal (B) and axial (C and D) chest CT scans revealed subcutaneous emphysema (red arrow), diffuse PM (green arrow), bilateral diffuse areas of ground-glass opacity, and consolidations (blue arrow).

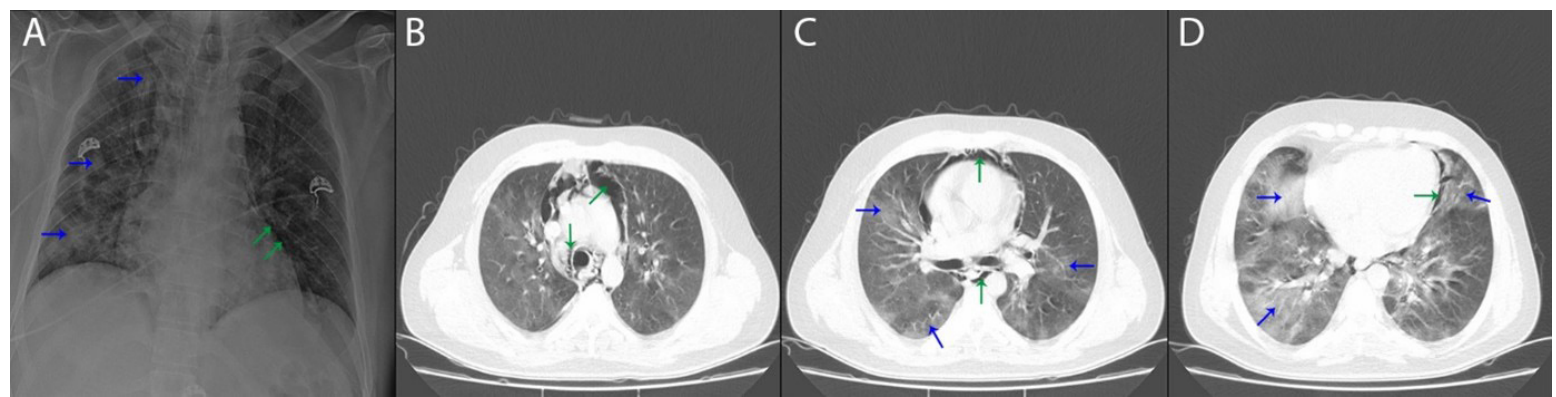

Figure 2: Radiological findings of Case 2. The posteroanterior chest radiograph (A) showed consolidations (blue arrow), and PM (green arrow). An axial (B, C and D) chest CT scans revealed diffuse PM (green arrow), bilateral diffuse areas of ground-glass opacity, and consolidations (blue arrow). 


\section{Discussion}

PM, which is defined as the presence of air in the mediastinum and is also referred to as mediastinal emphysema, is a rare condition that typically occurs secondary to trauma, pulmonary barotrauma, or mediastinal infections. Spontaneous PM is most often caused by increased airway pressure, secondary to mechanical ventilation or airway obstruction. The pathophysiology of spontaneous PM in COVID-19 patients is associated with increased distal airway pressure due to repeated coughing spells or the rupture of the alveolar membrane, secondary to diffuse alveolar membrane damage induced by the virus ${ }^{4}$. The symptoms of COVID-19 infection have been widely described, with the most frequent signs including high fever, chills, cough, dyspnea, diarrhea, myalgia, fatigue, expectoration, and hemoptysis ${ }^{5}$. Spontaneous PM associated with COVID-19 has occasionally been reported ${ }^{4,6-8}$. The management of spontaneous PM in the ED consists of the maintenance of respiratory integrity, hemodynamic stabilization, and the initiation of interventions designed to prevent further complications, such as tension PM, mediastinitis, airway obstruction, and pneumothorax. Both of our patients, whose RT-PCR COVID-19 tests were positive, presented with CT findings consistent with the advanced stages of COVID-19 infection. First patient's chest CT showed bilateral confluent areas of groundglass opacities and alveolar consolidation, PM, and subcutaneous emphysema (Figure 1). Similarly second patient's chest CT scan revealed diffuse PM, bilateral diffuse areas of ground-glass opacity, and consolidations, which are all features compatible with COVID-19 pneumonia (Figure 2). The probable cause of spontaneous PM and subcutaneous emphysema associated with COVID-19 in both of our cases was alveolar damage. Alveolar rupture can occur when a pressure gradient exists between the alveoli and the perivascular sheath. Therefore, in these cases, severe diffuse alveolar damage and episodes of coughing or Valsalva maneuver can lead to spontaneous PM. Subcutaneous emphysema detected on physical examination may occur due to the spread of extra-alveolar air to the neck, face, and anterior chest wall. In addition to subcutaneous emphysema, a physical examination may reveal a crackling sound synchronous with the heartbeat (Hamman's crunch), which is pathognomonic for PM. On chest X-ray of first case, we observed air in the mediastinum and free air in the soft tissues of the cervical area. These findings were consistent with PM and subcutaneous emphysema, respectively. Thoracic CT is the current gold standard for the diagnosis of PM. Bronchoscopy and esophagoscopy examinations can be utilized to distinguish possible ruptures in the bronchial tree and esophagus (Boerhaave's syndrome), respectively, to complement chest CT. Subcutaneous emphysema detected on physical examination in patients occurs due to the spread of extra-alveolar air to the neck, face, and anterior chest wall ${ }^{9,10}$. Spontaneous
PM and subcutaneous emphysema are not common for the clinical presentation of COVID-19 infection; however, these presentations can represent potentially aggravating factors during the management of COVID-19 pneumonia. Despite early diagnosis and optimal management, spontaneous PM associated with COVID-19 resulted in the death of our first patient. However, our second patient was discharged home.

\section{Conclusion}

Spontaneous PM is a rare complication of viral pneumonia that can progress from a mild clinical condition to a life-threatening clinical situation. Spontaneous PM and subcutaneous emphysema must be recognized as possible complications of COVID-19 infection and deserve early recognition. Even with a normal chest X-ray, a patient suspected of traumatic or spontaneous PM should undergo a CT scan. During the ongoing COVID-19 pandemic, physicians should approach the diagnosis and treatment of this complication with caution.

\section{References}

1. Huang C, Wang Y, Li X, Ren L, Zhao J, Hu Y, et al. Clinical features of patients infected with 2019 novel coronavirus in Wuhan, China. Lancet 2020; 395(10223): 497-506.

2. Shan $S$, Guangming $L$, Wei $L$, Xuedong Y. Spontaneous pneumomediastinum, pneumothorax and subcutaneous emphysema in COVID-19: case report and literature review. Rev Inst Med Trop São Paulo 2020; 62: e76.

3. Janssen J, Kamps MJA, Joosten TMB, Barten DG. Spontaneous pneumomediastinum in a male adult with COVID-19 pneumonia. Am J Emerg Med 2020; 30: S0735-6757(20)30661-6.

4. Nieves-Ortiz AA, Fonseca-Ferrer V, Hernández-Moya $K$, Ramirez $\mathrm{KM}$, Ayala-Rivera J, Delgado M. Spontaneous pneumomediastinum associated with COVID-19:Rare complication of 2020 pandemic. J Pulmonol Respir Res 2020; 4: 018-020.

5. Pal M, Berhanu G, Desalegn C, Kandi V. Severe Acute Respiratory Syndrome Coronavirus2 (SARS-CoV-2): An Update. Cureus 2020; 12(3): e7423.

6. Zhou C, Gao C, Xie Y, Xu M. COVID-19 with spontaneous pneumomediastinum. Lancet Infect Dis 2020; 20(4): 510.

7. Mohan V, Tauseen RA. Spontaneous pneumomediastinum in COVID-19. BMJ Case Rep 2020; 13: e236519.

8. Ucpinar BA, Sahin C, Yanc U. Spontaneous pneumothorax and subcutaneous emphysema in COVID-19 patient: case report. J Infect Public Health 2020; 13: 887-9.

9. Mansella $\mathrm{G}$, Bingisser $\mathrm{R}$, Nickel $\mathrm{CH}$. Pneumomediastinum in blunt chest trauma: a case report and review of the literature. Case Rep Emerg Med 2014; 2014: 685381.

10. Brogna B, Bignardi E, Salvatore P, Alberigo M, Brogna C, Megliola A. Unusual presentations of COVID-19 pneumonia on CT scans with spontaneous pneumomediastinum and loculated pneumothorax: A report of two cases and a review of the literature. Heart Lung 2020; 49(6): 864-8. 